\title{
On-Pump Coronary Artery Bypass Grafting in a Patient With Renal Transplant and Familial Hypercholesterolemia
}

\author{
Fatih Aygun
}

\begin{abstract}
A 19-year-old male patient presented to the cardiology clinic with complaints of chest pain during efforts for a month. He had a renal transplantation when he was 12 years old. He had been diagnosed with familial hypercholesterolemia. On-pump coronary artery bypass grafting (CABG) surgery (LIMA-LAD, DI, Cx (OMI), RCA (PD)) was performed. On-pump CABG can be performed successfully in patient with renal transplant. However, renal recipients have higher risk of infection and renal damage compared with patients without transplants. In addition, as in our case, familial hypercholesterolemia would affect the clinical outcome negatively. I would like to think the sensitivity and close follow-up in standard CABG cases, and it should be applied to the highest level in such patients.
\end{abstract}

Keywords: Renal transplantation; Familial hypercholesterolemia; Coronary atherosclerosis; Coronary artery bypass grafting

\section{Introduction}

Coronary artery bypass grafting (CABG) is frequently used worldwide. Coronary artery bypass operation is performed while the heart is stopped and using cardiopulmonary bypass machine is called on-pump CABG [1]. The surgical procedure involves renal, cardiac, cerebral, and respiratory risks [2].

Familial hypercholesterolemia (FH) is an autosomal dominant disease, which is characterized by high total cholesterol (TC) and low-density lipoprotein (LDL) cholesterol levels from birth. It is a major cause of premature coronary heart disease, and needs to be treated with CABG surgery at an early age [3].

Renal transplantation is a form of treatment in patients with end-stage renal disease [4]. The immunosuppressive drugs used after kidney transplantation increase the risk of CABG surgery.

We present a different case in our clinic, in which on-pump $\mathrm{CABG}$ was performed in a young patient with renal transplant

Manuscript submitted July 14, 2017, accepted July 25, 2017

Baskent University, Konya Medical and Research Center, Cardiovascular Surgery, Konya, Turkey. Email: drfatihaygun@gmail.com

doi: https://doi.org/10.14740/jmc2878w and had diagnosed hypercholesterolemia.

\section{Case Report}

A 19-year-old male patient presented to the cardiology clinic with complaints of chest pain during efforts for a month. He had a history of renal transplantation when he was 12 years old. He had no history of diabetes mellitus, hypertension, smoking and family history of coronary artery disease. He had been diagnosed with FH. He has taken the immunosuppressive drug cyclosporin (SANDIMMUN ${ }^{\circledR}$ Neoral $25 \mathrm{mg}$ ) and mycophenolatmofetil (Cellcept ${ }^{\circledR} 500 \mathrm{mg}$ ) due to renal transplantation and statin (Alvastin ${ }^{\circledR} 80 \mathrm{mg}$ ) due to hyperlipidemia.

His physical examination results showed no abnormalities. Vital findings were within normal range: fever $36.7^{\circ} \mathrm{C}$, blood pressure arterial $110 / 60 \mathrm{~mm} \mathrm{Hg}$ with a regular pulse of 74 beats/min, respiration rate $14 / \mathrm{min}$, and saturation with $94 \% / 96 \%$ with pulse oximeter. In his physical examination, heart sounds were rhythmic and no additional sound murmur was detected. All peripheral pulses were palpable. Both hemithoraxes participated in respiration equally, and there was no ral-rhonchus. There was no distension, rebound or tenderness in abdomen. In local examination, there was the renal transplantation scar.

On laboratory investigation, cardiac markers were normal; GFR $79 \mathrm{~mL} / \mathrm{min} / 1.73 \mathrm{~m}^{2}$ (normal range $>60$ ), blood urine nitrogen $20 \mathrm{mg} / \mathrm{dL}$, creatinine $1.3 \mathrm{mg} / \mathrm{dL}$, serum cyclosporin level $77 \mathrm{ng} / \mathrm{mL}$, serum total cholesterol $596 \mathrm{mg} / \mathrm{dL}$ (normal range $130-200 \mathrm{mg} / \mathrm{dL}$ ), HDL cholesterol $42 \mathrm{mg} / \mathrm{dL}$ (normal range $130-200 \mathrm{mg} / \mathrm{dL}$ ), LDL cholesterol $533 \mathrm{mg} / \mathrm{dL}$ (normal range $60-130 \mathrm{mg} / \mathrm{dL}$ ), and triglyceride 106 (normal range 50 - 160 $\mathrm{mg} / \mathrm{dL}$ ) were determined.

An electrocardiogram showed ST-T changes (common ST depression). Echocardiography showed normal ventricular function with no cardiac valve pathology. Coronary angiogram revealed over $75 \%$ stenosis at the proximal left anterior descending artery (LAD) (Fig. 1), Cx coronary artery (Fig. 2) and right coronary artery (Fig. 3 ).

The patient was referred to cardiovascular surgery clinic for $C A B G$ surgery. The immunosuppressive and statin drugs were used until operation day. On-pump CABG surgery (LIMA-LAD, DI, Cx (OMI), and RCA (PD)) was performed. Because of the posssibility of renal failure that led to requirement of hemodialysis and AV fistula, safen vein conduit was preferred instead of radial vein. At the end of the operation, low-dose dopamine $(3 \mu \mathrm{g} / \mathrm{kg} / \mathrm{dk})$ and nitroglycerin infusion 


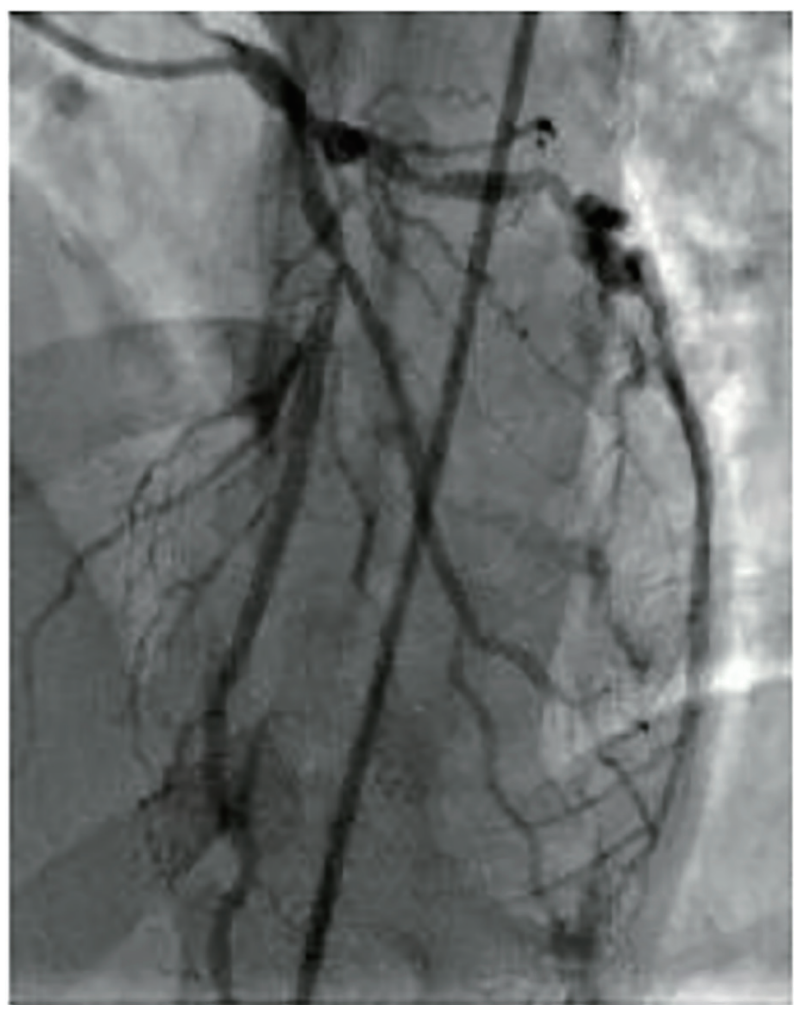

Figure 1. LAD lesion.

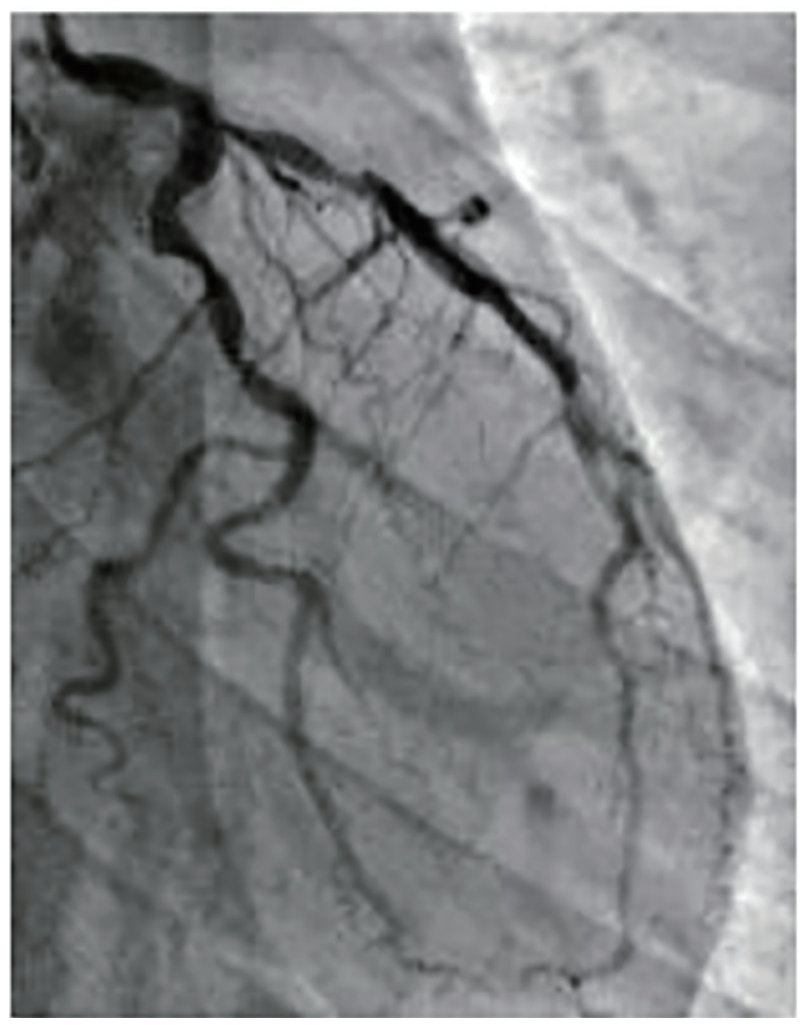

Figure 2. Cx lesion.

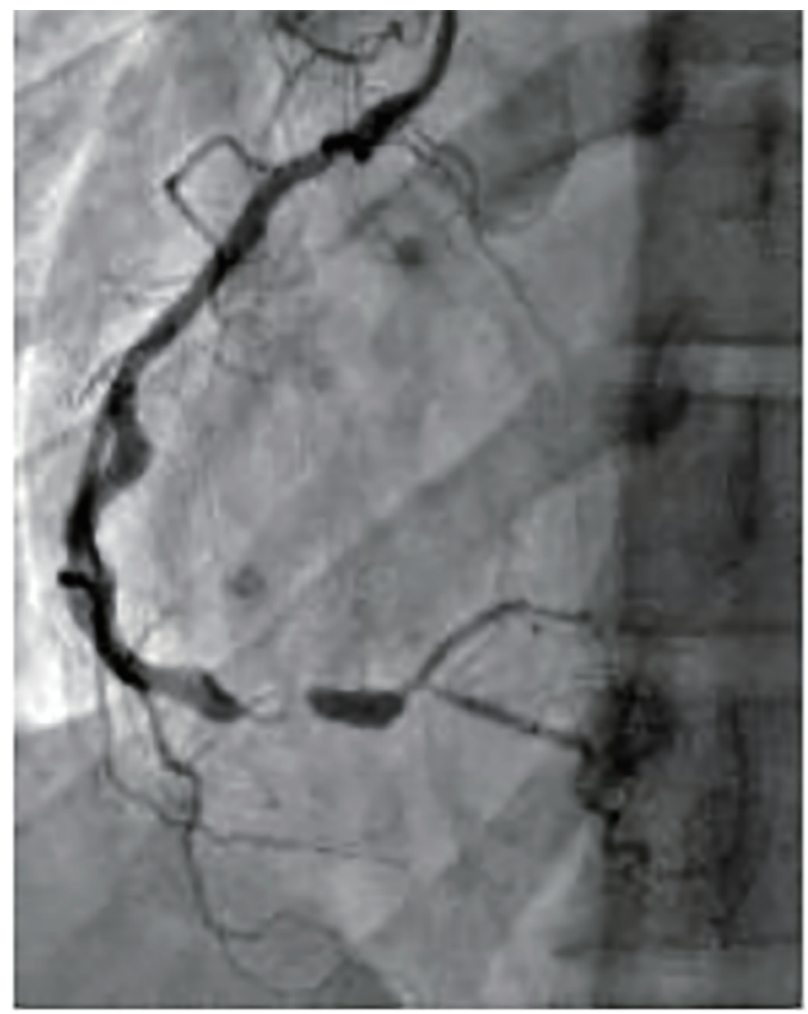

Figure 3. RCA lesion.

were given and patient was taken to ICU. Because of the fast-track anesthesia, patient was early extubated. Mobilization on the first postoperative day was achieved. Drains were removed on second postoperative day. After routine $\mathrm{CABG}$ follow-up, he was discharged on eighth day postoperatively. The last follow-up was 5 months after the surgery and he was remarkably well.

\section{Discussion}

CABG is a cardiac surgery procedure which is usually applied over 50 years of age. Although risks and benefits are individualized and depend on surgical technique, cerebrovascular events and renal insufficiency are main complications [5].

FH is a genetic disorder that clinically leads to an increase in plasma cholesterol levels. It increases three- to six-fold in homozygous carriers of FH mutations and two- to three-fold in heterozygous. FH patients often have coronary artery disease at second and third decades of their life; however, a 7-year-old FH patient had CABG as reported in the literature [6].

Development of atherosclerotic plaque deposition may also occur in the aorta at an early age in patients with $\mathrm{FH}$, as in our case. Therefore, we think that importance of the good examination of the aorta during aortic cannulation in CABG operations should be emphasized in patient with FH. My opinion is that it is getting more value of cannulation in the appropriate area and the side clamping in once at the clean area in the patients that aortic plaque placement is probable just like 
our case who has aortic soft plaque.

We also thought that in our case, 19-year-old male undergoing renal transplantation, the $\mathrm{CABG}$ operation has a higher risk of renal damage compared to the normal individual. In literature, data on the long-term outcomes and risk factors in this population are scarce; however, transient deterioration of graft function has been reported. In our case, as the patient had a history of renal transplantation, and is administered immunosuppressants, he is susceptible to infection or rejection.

In our case, as the patient was in the acute phase after renal transplantation, prevention of rejection was thought to be of the utmost importance. Moreover, the use of cardiopulmonary bypass $(\mathrm{CPB})$ in open heart surgery causes renal function and levels of immunosuppressants to become unstable. In our case, the particular attention is focused on the method used for administering immunosuppressants to this patient perioperatively. Preoperative immunosuppression which continued until the morning of the surgery was restarted as soon as possible postoperatively. Drugs was administered orally the night after surgery.

Immunosuppressive agents taken until operation day and its starting to take medicine as soon as possible after extubation are good managements for prevention from rejection. Their negative effect on renal function cannot be denied. Attention is paid to hypervolemia and hyperpotassemia in patients undergoing standard on-pump CABG. However, in such patients with kidney transplant and $\mathrm{FH}$, more care should be taken. I think that do not hesitate performing intraoperative ultrafiltration just as we did.

\section{Conclusion}

On-pump CABG can be performed succesfully in patient with renal transplant. However, renal recipients have higher risk of infection and renal damage compared with patients without transplants. In addition, as in our case, FH would affect the clinical outcome negatively.

Our precautions to be taken into consideration to prevent renal damage and infection for the patient with a history of renal transplantation include mediastinal washing, achieving adequate hemostasis, intraoperative ultrafitration, using blood product as little as possible, for protection of infection avoid- ing unnecessary cautery, and multidisciplinary approach with nephrology department is also important to manage the treatment especially in perioperative period.

I would like to think the sensitivity and close follow-up in standard CABG cases, and it should be applied to the highest level in such patients.

\section{Conflict of Interest}

None.

\section{References}

1. Lassnigg A, Hiesmayr MJ, Bauer P, Haisjackl M. Workgroup on Postoperative Intensive Care of the European Society of Intensive Care M, European Workgroup of Cardiothoracic I. Effect of centre-, patient- and procedurerelated factors on intensive care resource utilisation after cardiac surgery. Intensive Care Med. 2002;28(10):14531461.

2. Levy JH, Tanaka KA. Inflammatory response to cardiopulmonary bypass. Ann Thorac Surg. 2003;75(2):S715720.

3. Kawasuji M, Sakakibara N, Takemura H, Matsumoto Y, Mabuchi H, Watanabe Y. Coronary artery bypass grafting in familial hypercholesterolemia. J Thorac Cardiovasc Surg. 1995;109(2):364-369.

4. Magee CC, Pascual M. Update in renal transplantation. Arch Intern Med. 2004;164(13):1373-1388.

5. Hillis LD, Smith PK, Anderson JL, Bittl JA, Bridges CR, Byrne JG, Cigarroa JE, et al. 2011 ACCF/AHA Guideline for Coronary Artery Bypass Graft Surgery. A report of the American College of Cardiology Foundation/American Heart Association Task Force on Practice Guidelines. Developed in collaboration with the American Association for Thoracic Surgery, Society of Cardiovascular Anesthesiologists, and Society of Thoracic Surgeons. J Am Coll Cardiol. 2011;58(24):e123-210.

6. Ersoy U, Guvener M. Coronary revascularization in seven-year-old boy with homozygous familial hypercholesterolaemia. Acta Paediatr. 2000;89(12):1501-1502. 